\title{
Where can the water go? Giant impacts and the deep mantle
}

\section{SETH A JACOBSON}

\author{
Michigan State University
}

Presenting Author: seth@msu.edu

Water is accreted by the terrestrial planets throughout their formation history, however delivery of water is expected to increase near the end of accretion due to radial mixing from the outer parts of the terrestrial disk bringing in ice-rich, C-type planetesimals [1]. Even in the context of pebble accretion, as the water ice-line moves inward, water delivery will increase with time. Generally, accretion builds up planets analogously to sediments in a basin, individual impacts are local events and the most recent accreted material gets mixed into only the topmost layers. However, giant impacts deliver enough energy to melt significant portions of a planet and to significant depth [2]. Within these post-giant impact mantle magma oceans, water is soluble and mixed throughout the entire magma ocean by the expected active convection. Here, we show how the giant impact accretion history of terrestrial planets determines the location of final water reservoirs within a planet. Planets like Earth with late giant impacts have water mixed deep into their interiors. This water may be trapped in the deep interior if magma ocean solidification is not bottom-up, potentially even extending the lifetime of a putative basal magma ocean dynamo. Water mixed to transition zone depths would create hydrated minerals during magma ocean solidification, changing the viscous and elastic properties of the mid-mantle and precluding the need for subduction to "wet" the upper mantle and asthenosphere. Alternatively, planets without late giant impacts, as hypothesized for Venus [3], would not have late and deep mantle magma oceans. Instead, water in these planets would preferentially be found in the uppermost accreted layers. Furthermore, water delivered in each impact event would likely either be immediately vaporized into the atmosphere or quickly degassed from a small melt pool during its crystallization. While giant impacts may not deliver the water themselves, they are crucial for determining where that water ends up in the planet at the end of accretion.

[1] e.g. O'Brien et al. (2018), SSR 214 (1), 47.

[2] e.g. Nakajima \& Stevenson (2015), EPSL 427, 286-295.

[3] Jacobson et al. (2017), EPSL 474, 375-386. 